\title{
Open charm measurements at CERN SPS energies in the NA61/SHINE experiment - status and plans
}

\author{
Anastasia Merzlaya for the NA61/SHINE Collaboration* \\ Jagiellonian University, Saint Petersburg State University \\ E-mail: anastasia.merzlayaecern.ch
}

\begin{abstract}
The study of open charm meson production provides an efficient tool for detailed investigations of the properties of hot and dense matter formed in nucleus-nucleus collisions. In particular, charm mesons are of vivid interest in the context of the phase-transition between confined hadronic matter and the quark-gluon plasma. Recently, the experimental setup of NA61/SHINE experiment was supplemented with a small-acceptance version of the Vertex Detector (SAVD) which was motivated by the importance and the possibility of the first direct measurements of open charm mesons in heavy ion collisions at SPS energies. First exploratory data taking of $\mathrm{Pb}+\mathrm{Pb}$ collisions at $150 \mathrm{~A} \mathrm{GeV/c}$ with SAVD was performed in 2016, and a $D^{0}$ signal was extracted in it's $D^{0} \rightarrow \pi^{+}+K^{-}$decay channel. This was the first, direct observation of open charm in nucleusnucleus collisions at the SPS energies. In 2017 and 2018 a large statistic data set has been taken for $\mathrm{Xe}+\mathrm{La}$ and $\mathrm{Pb}+\mathrm{Pb}$ with $\mathrm{SAVD}$ at $150 \mathrm{~A} \mathrm{GeV/c}$; these data are currently under intense analysis. Furthermore, the future plans of open charm measurements in the NA61/SHINE experiment related to the upgraded version of the Vertex Detector are discussed.
\end{abstract}

Corfu Summer Institute 2018 "School and Workshops on Elementary Particle Physics and Gravity" (CORFU2018)

31 August - 28 September, 2018

Corfu, Greece

${ }^{*}$ Speaker. 


\section{Introduction}

One of the important issues related to relativistic heavy-ion collisions is the mechanism of charm production. Several models were developed to describe charm production. Some of them are based on the dynamical approach and some on the statistical approach. The estimates from these approaches for the average number of produced $c$ and $\bar{c}$ pairs $(\langle c \bar{c}\rangle)$ for central $\mathrm{Pb}+\mathrm{Pb}$ collisions at $158 \mathrm{~A} \mathrm{GeV/c}$ differ by up to a factor of $50[1,2]$ which is illustrated in Figure 1. Therefore, obtaining precise data on $\langle c \bar{c}\rangle$ will allow discriminating between theoretical models and learning about the charm quark and hadron production mechanism.

A good estimate of $\langle c \bar{c}\rangle$ can be obtained by measuring production yields of $D^{0}, D^{+}$, and their antiparticles because these mesons carry about $85 \%$ of the total produced charm for central $\mathrm{Pb}+\mathrm{Pb}$ collisions at $150 \mathrm{~A} \mathrm{GeV/c}$ as it is shown in Figure 2 (this value is predicted by the Parton Hadron String Dynamics (PHSD) model [9]).

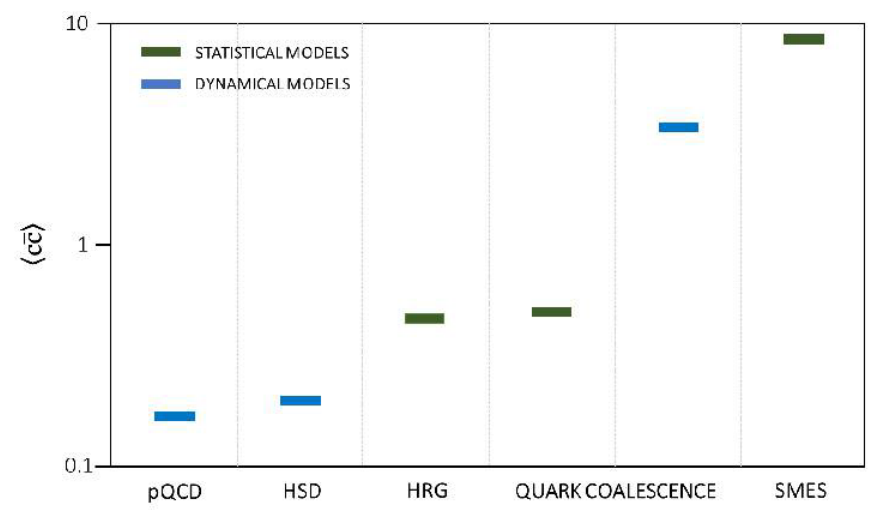

Figure 1: Mean multiplicity of charm quark pairs produced in the full phase space in central $\mathrm{Pb}+\mathrm{Pb}$ collisions at $158 \mathrm{~A} \mathrm{GeV/c}$ calculated with dynamical models (blue bars): HSD [3, 4], pQCD-inspired $[5,6]$, and Dynamical Quark Coalescence [7], as well as statistical models (green bars): HRG [8], Statistical Quark Coalescence [8] and SMES [2].

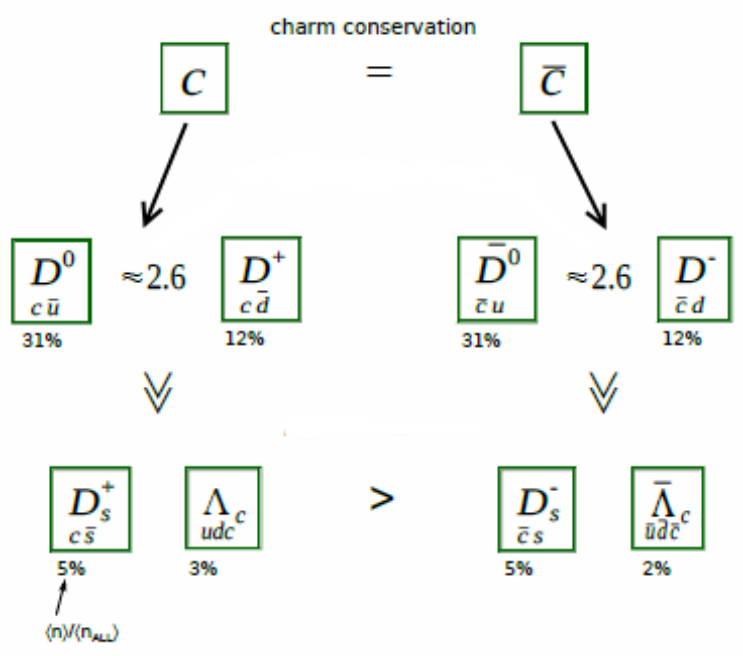

Figure 2: Distribution of total produced charm in open charm particles for central $\mathrm{Pb}+\mathrm{Pb}$ collisions at $150 \mathrm{~A} \mathrm{GeV/c}$ as predicted by the PHSD model.

The study of open charm meson production is a sensitive tool for detailed investigations of the properties of hot and dense matter formed in nucleus-nucleus collisions at relativistic energies $[10,11,12]$. In particular, charm mesons are of special interest in the context of the phase-transition between confined hadronic matter and the Quark-Gluon Plasma (QGP). 
The $c \bar{c}$ pairs produced in the collisions are converted into open charm mesons and charmonia ( $\mathrm{J} / \psi$ mesons and their excited states). The production of charm is expected to be different in confined and deconfined matter. This is caused by different properties of charm carriers in these phases. In confined matter, the lightest charm carriers are $D$ mesons, whereas in deconfined matter, the lightest carriers are charm quarks. Production of a $D \bar{D}$ pair $\left(2 \mathrm{~m}_{D}=3.7 \mathrm{GeV}\right)$ requires an energy about $1 \mathrm{GeV}$ higher than the production of a the $c \bar{c}$ pair $\left(2 \mathrm{~m}_{c}=2.6 \mathrm{GeV}\right)$. The effective number of degrees of freedom of charm hadrons and charm quarks is similar [13]. Thus, more abundant charm production is expected in deconfined than in confined matter. Consequently, in analogy to strangeness $[2,14]$, a change of the collision energy dependence of the $\langle c \bar{c}\rangle$ yield may be a signal of the onset of deconfinement.

Up to now, direct measurements of open charm had never been performed at the Super Proton Synchrotron (SPS) energies. The first estimate of the upper limit of mean multiplicity of $D$ and $\bar{D}$ mesons by a direct measurement was done by the NA49 experiment in $\mathrm{Pb}+\mathrm{Pb}$ collisions at the top SPS energies [15].

The NA38/NA50 and NA60 experiments at the European Organization for Nuclear Research (CERN) provided measurements of $\mathrm{J} / \psi$ production at the top SPS energy in the di-muon decay channel $[16,17]$. In Figure 3 the production of $\mathrm{J} / \psi$ in $\mathrm{In}+\mathrm{In}$ and $\mathrm{Pb}+\mathrm{Pb}$ collisions is shown as a function of the number of participating nucleons relative to the perturbative Quantum Chromodynamics (pQCD) predictions (assuming normal nuclear absorption in the medium). For a lower number of participants, the yields are consistent with the theoretical estimations. However, at $N_{\text {part }}>200$, the result shows a significant drop. This so called anomalous suppression was attributed to the $\mathrm{J} / \psi$ dissociation effect in the deconfined medium $[18,10]$. However, due to initial state effects in nucleus-nucleus reactions, like shadowing, parton energy loss, etc. [19], the overall scaled number of the $c \bar{c}$ pairs produced in nuclear collisions may be reduced. Thus this effect can only be determined by studying the charmonium yield relative to the total charm yield, which can be determined by measuring open charm yields [11].

The probability of a $c \bar{c}$ pair hadronizing into a $\mathrm{J} / \psi$ meson is defined as $P(c \bar{c} \rightarrow J / \psi) \equiv \frac{\langle J / \psi\rangle}{\langle c \bar{c}\rangle} \equiv$ $\frac{\sigma_{J / \psi}}{\sigma_{c \bar{c}}}$. To be able to determine this probability, one needs data on both $\mathrm{J} / \psi$ and $c \bar{c}$ yields in the full phase space. This can be done only by studying the charmonium yield relative to the yield of open charm mesons [11].

Thus, systematic measurements of open charm production are urgently needed for the interpretation of the existing results on $\mathrm{J} / \psi$ suppression in a model independent way. Such measurements would allow disentangling initial and final state effects, revealing the properties of hidden and open charm transport through the dense medium created in nucleus-nucleus collisions.

Figure 4 shows present and future facilities and their region of coverage in the phase diagram of strongly-interacting matter. However, not all of them perform/plan measurements of charm hadrons in nucleus-nucleus collisions:

- The Large Hadron Collider (LHC) and the Relativistic Heavy Ion Collider (RHIC) at high energies $\left(\sqrt{s_{N N}} \gtrsim 200 \mathrm{GeV}\right)$ : measurements of charm particles are performed in an acceptance limited to mid-rapidity; this limitation is due to the collider kinematics and related to 


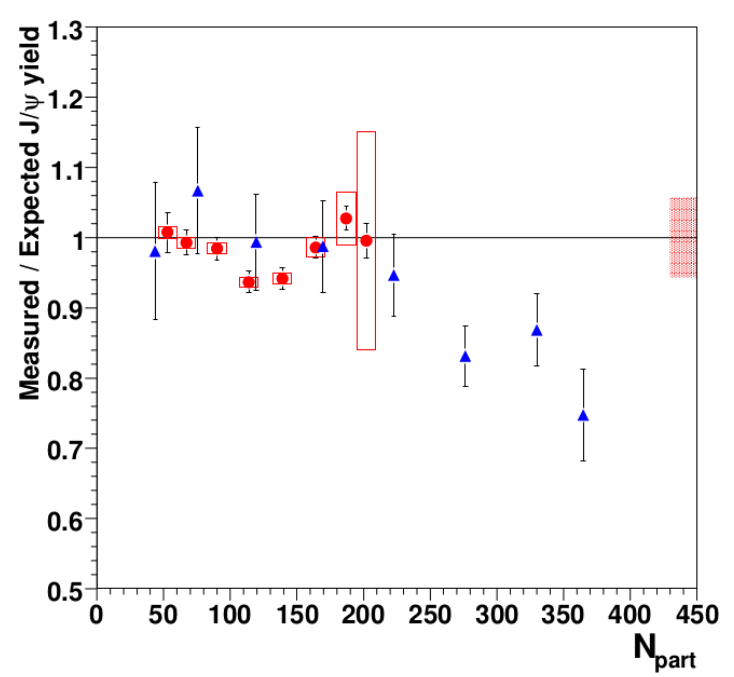

Figure 3: $\mathrm{J} / \psi$ suppression pattern measured in In + In (red dots) and $\mathrm{Pb}+\mathrm{Pb}$ (blue triangles) collisions as a function of the number of participants at the top Super Proton Synchrotron (SPS) energy [16, 17]. The boxes around the In + In points represent correlated systematic errors. The shaded box on the right shows the uncertainty in the absolute normalization of the $\mathrm{J} / \psi$ yields for the In + In interactions.

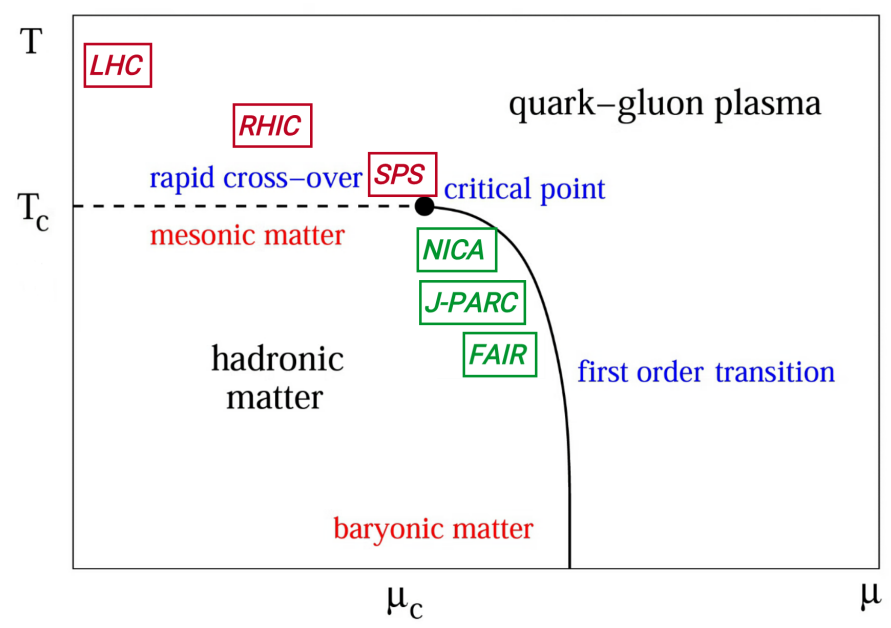

Figure 4: Regions in the phase diagram of strongly-interacting matter explored by present (red) and future (green) heavy ion facilities. RHIC, Relativistic Heavy Ion Collider; NICA, Nuclotron-based Ion Collider fAcility; J-PARC, Japan Proton Accelerator Research Complex Heavy-Ion project; FAIR, Facility for Antiproton and Ion Research.

the detector geometry [20, 21, 22, 23]. At very high energy collisions, the multiplicities of $c \bar{c}$ pairs are high, which may lead to the secondary formation of $\mathrm{J} / \psi$ mesons, complicating the study of the in-medium effect on $\mathrm{J} / \psi$ meson production;

- The RHIC Beam Energy Scan (BES) collider program $\left(\sqrt{s_{N N}}=7.7-39 \mathrm{GeV}\right)$ : measurement is not considered in the current program; this may likely be due to difficulties related to collider geometry and kinematics, as well as the low charm production cross-section [24, 25];

- The RHIC BES fixed-target program $\left(\sqrt{s_{N N}}=3-7.7 \mathrm{GeV}\right)$ : not considered in the current program [26];

- The Nuclotron-based Ion Collider fAcility (NICA) $\left(\sqrt{s_{N N}}<11 \mathrm{GeV}\right)$ : measurements during Stage 2 (after 2023) are considered [27];

- The Japan Proton Accelerator Research Complex Heavy-Ion project (J-PARC-HI) $\left(\sqrt{s_{N N}} \lesssim\right.$ $6 \mathrm{GeV}$ ): under consideration; might be possible after 2025 [28]; 
- The Facility for Antiproton and Ion Research (FAIR) Schwer-Ionen-Synchrotron (SIS-100) $\left(\sqrt{s_{N N}} \lesssim 5 \mathrm{GeV}\right)$ : measurements of charm hadrons are not possible due to the very low crosssection at SIS-100, systematic charm measurements are planned with SIS-300 $\left(\sqrt{s_{N N}} \lesssim\right.$ $7 \mathrm{GeV}$ ), which is part of the FAIR project; however, no time estimation is available [29].

The conclusion is that only NA61/SHINE will be able to measure open charm production in heavy ion collisions in full phase space in the near future. The corresponding potential measurements at higher (LHC, RHIC) and lower (FAIR, J-PARC) energies are necessary to complement the NA61/SHINE results and establish by collisions the energy dependence of charm production.

\section{NA61/SHINE Experiment for Open Charm Measurements}

\subsection{NA61/SHINE Facility}

The SPS Heavy Ion and Neutrino Experiment (NA61/SHINE) [30] at CERN was designed for studies of the properties of the onset of deconfinement and the search for the critical point of strongly-interacting matter. These goals are being pursued by investigating $\mathrm{p}+\mathrm{p}, \mathrm{p}+\mathrm{A}$, and $\mathrm{A}+$ A collisions at different beam momenta from $13 A-158 A \mathrm{GeV} / c$ for ions and up to $400 \mathrm{GeV} / c$ for protons. It is a fixed target experiment, which is better suited than a typical collider experiment for the detection of strange and multi-strange particles, as well as heavy flavors, like charmed particles. The layout of the experimental setup is shown in Figure 5.

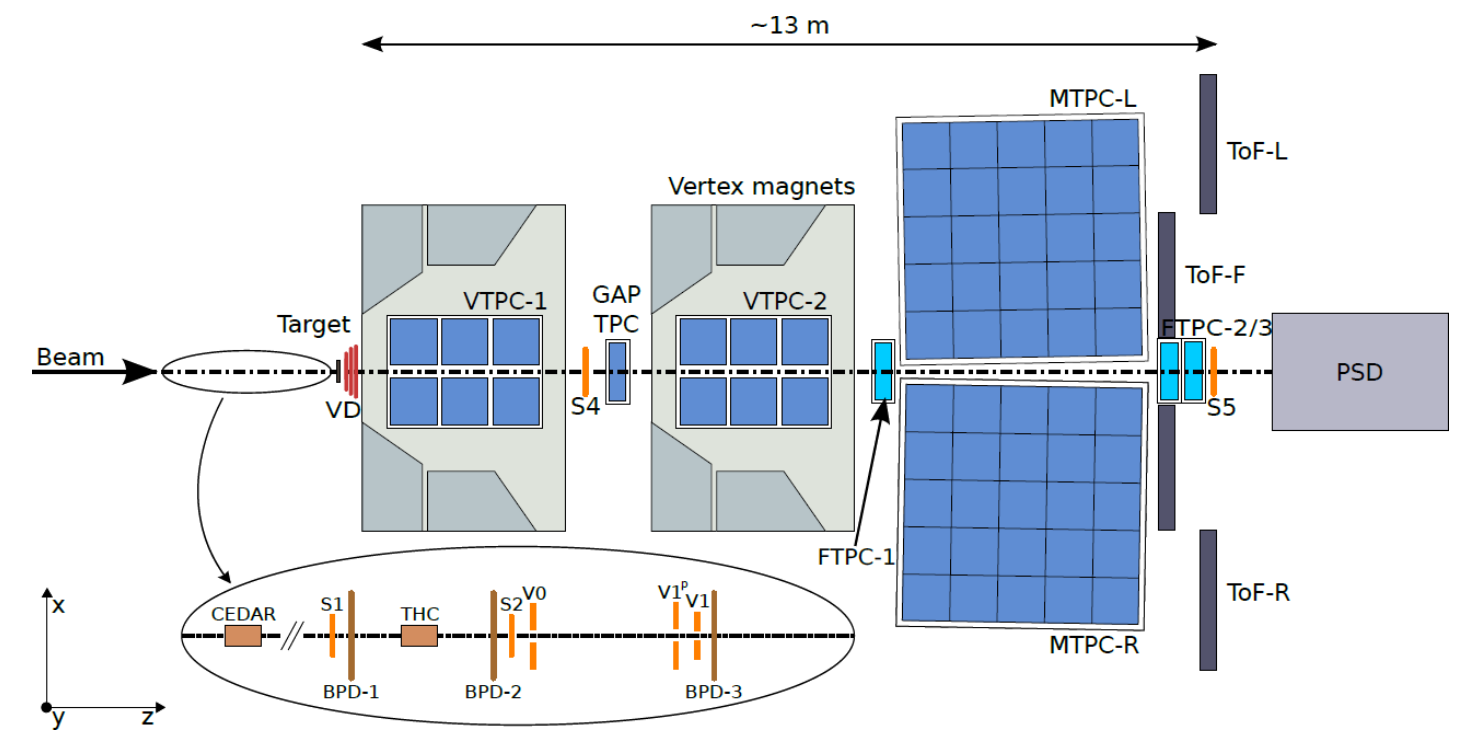

Figure 5: Layout of the NA61/SHINE experimental setup (top view, not to scale). VTPC, Vertex Time Projection Chamber; MTPC, Main TPC; PSD, Projectile Spectator Detector; BPD, Beam Position Detector.

The main component of the facility is the large acceptance hadron spectrometer, which was inherited from the NA49 experiment [31]. The setup includes the Beam Position Detectors (BPD), Cherenkov counters, and the scintillator detectors located upstream of the target. They provide information on the timing, charge, and position of beam particles. Charged particle tracking is provided by two Vertex Time Projection Chambers (VTPC-1 and VTPC-2), which are located 
inside magnets, the gap TPC, and two Main TPCs (MTPC-L on the left side of the beam line and MTPC-R on the right side). These TPCs provide acceptance in the full forward hemisphere, down to $p_{T}=0$. The TPCs allow for momentum and charge reconstruction, as well as measurement of mean energy loss per unit path length, which is used for particle identification. The Time-of-Fight (ToF) walls, which are used to improve particle identification, are located behind the main TPCs. The Projectile Spectator Detector (PSD) measures the energy of projectile spectators and delivers information on collision centrality.

\subsubsection{Vertex Detector in NA61/SHINE}

For open charm measurements in nucleus-nucleus collisions, NA61/SHINE was upgraded with the new Small Acceptance version of the Vertex Detector (SAVD).

As was already mentioned, open charm mesons are difficult to measure because of their low yields and short lifetime. These mesons can in principle be detected in their decay channels into pions and kaons. However, in heavy ion collisions, $\pi \mathrm{s}$ and $K \mathrm{~s}$ are produced in large numbers by other sources, giving a huge background in the invariant mass distributions (see Figure 6). To distinguish the daughter particles of $D^{0}$ mesons from hadrons produced at the primary nucleus-nucleus interaction point, one aims to select only hadron pairs generated in a secondary decay vertex. Vertex reconstruction is done by extrapolating the trajectories back to the target and identifying intersection points. The primary vertex will typically appear as the intersection point of multiple tracks, while the tracks originating from weak decays will intersect at a well-defined displaced point (secondary vertex).

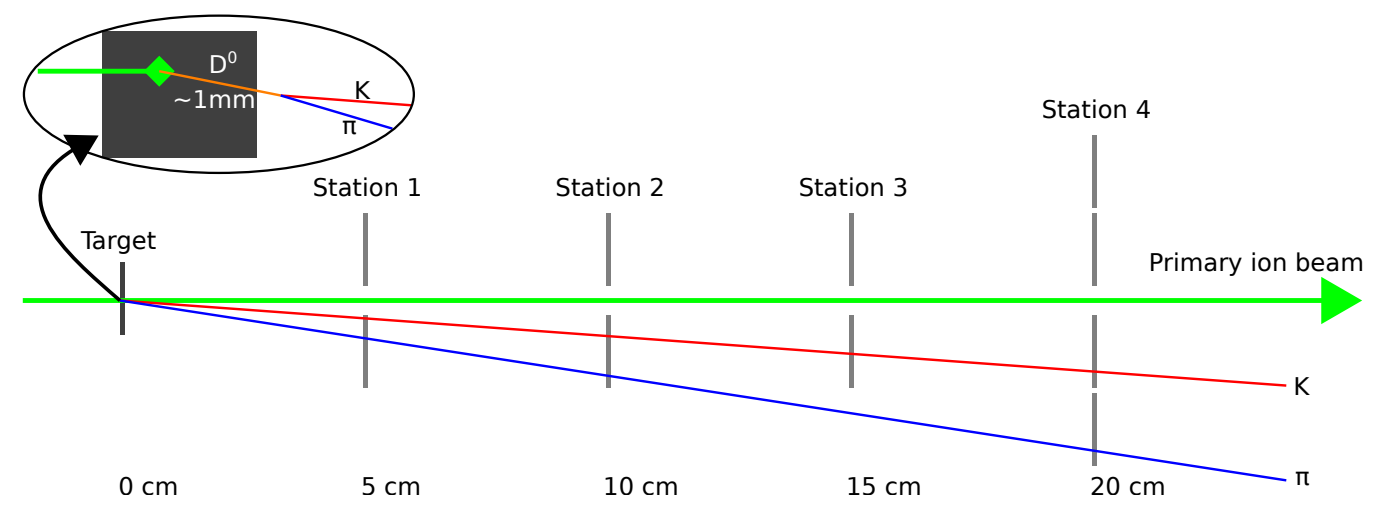

Figure 6: Schematics of the reconstruction strategy of the $D^{0} \rightarrow \pi^{+}+K^{-}$decay channel with the help of the SAVD.

The Vertex Detector was designed to perform high efficiency tracking and high resolution primary and secondary vertex reconstruction. The detector concept was developed in simulations $[32,33,34]$, and it only partially covers the total geometrical acceptance of NA61/SHINE. The simulations performed using the A Multi-Phase Transport Model (AMPT) event generator [35] as input show that about $5 \%$ of all $D^{0}+\overline{D^{0}}$ in the $\pi$ and $K$ decay channel can be registered by the SAVD and pass background suppression and quality cuts (see the details below).

The SAVD is positioned between the target and the VTPC1 (see Figure 5). It consists of two spectrometer arms located on left and right side of the beam, each composed of four detector 
planes (stations) of coordinate-sensitive detectors located at a 5,10,15, and $20 \mathrm{~cm}$ distance from the target. Each arm hosts in total eight sensors (see Figure 7).

High position resolution silicon Minimum Ionizing MOnolithic Active pixel sensors (MIMOSA26) [36] based on the complementary metal-oxide-semiconductor (CMOS) technology were chosen as the basic detection element of the SAVD stations. The pixel pitch is $18.4 \mu \mathrm{m}$ in each direction, which leads to a high spatial resolution of $3.5 \mu \mathrm{m}$. The sensors have a very low material budget (50 $\mu \mathrm{m}$ thickness), which minimizes the multiple scattering effect in the detection planes. All columns of the sensor are read out in parallel in the rolling shutter mode, and the readout time resolution is $115.2 \mu \mathrm{s}$, which is sufficient for data taking at collision rates $<1 \mathrm{kHz}$.

The MIMOSA-26 sensors are mounted on extra light-weight vertical carbon fiber ladders, developed for the upgrade of the Inner Tracking System (ITS) at the A Large Ion Collider Experiment (ALICE) [37]. The ladders are held by C-shaped support frames as illustrated in Figure 5 and have integrated pipes for water cooling. Further, the SAVD and the target were placed in a helium enclosure (aquarium) to minimize the multiple scattering of beam ions and produced particles. Because the detector is located close to the edge of the VTPC-1 magnet, the magnetic field in the SAVD volume is small and inhomogeneous $(0.13-0.25 \mathrm{~T})$.

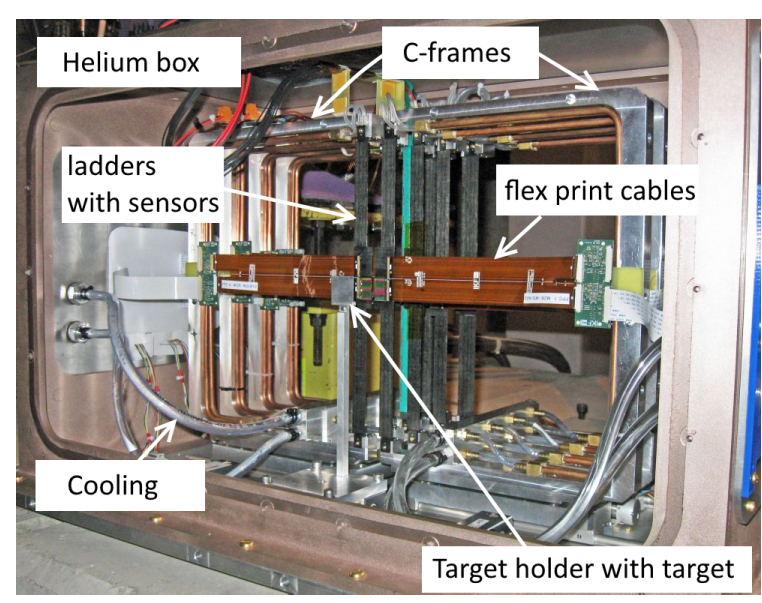

Figure 7: Photograph of the Small Acceptance version of the Vertex Detector (SAVD) before closing the detector with the front and exit windows. The detector elements are indicated.

The sensors are connected to front-end boards that provide sensor biasing. The front-end boards are connected to the data processing Trigger Readout Board (TRB) [38] developed for the updated Micro Vertex Detector (MVD) at the Compressed Baryonic Matter experiment (CBM) [39], which are responsible for the fast and slow control of the sensors.

\section{Open Charm Measurements with the SAVD}

\subsection{Data Reconstruction in the Vertex Detector}

The track finding algorithm is based on a combinatorial method (for pre-tracking) and the Hough transform algorithm (details are discussed in [40]). Primary vertex reconstruction was done by extrapolating the trajectories back to the target and identifying intersection points. The primary vertex will typically appear as the intersection point of multiple tracks, while the tracks originating from secondary decays will intersect at a well-defined displaced point (secondary vertex). The distribution of the longitudinal coordinate $\left(z_{\text {prim }}\right)$ of the primary vertex is shown in Figure 8 for 
$\mathrm{Xe}+\mathrm{La}$ data set. The structure of the La target, which was composed of three $1 \mathrm{~mm}$ plates, can be well seen on this distribution plot. A spatial primary vertex resolution of $\sigma_{x, y, z}=5,1.5,30 \mu \mathrm{m}$ has been achieved for test $\mathrm{Pb}+\mathrm{Pb}$ data taken in 2016. For the $\mathrm{Xe}+\mathrm{La}$ data, the resolution was found to be $\sigma_{x, y, z}=1.3,1.0,15 \mu \mathrm{m}$. The significant improvement compared to the pilot measurement was due to better setting of the sensor thresholds, resulting in increased pixel efficiency.

Figure 8: Distribution of the longitudinal coordinate $z_{\text {prim }}$ of primary vertices for tracks produced in the interactions in the La target, which was composed of three $1 \mathrm{~mm}$ plates. Plot is done for $\mathrm{Xe}+\mathrm{La}$ at $150 \mathrm{~A} \mathrm{GeV/c}$ data set.

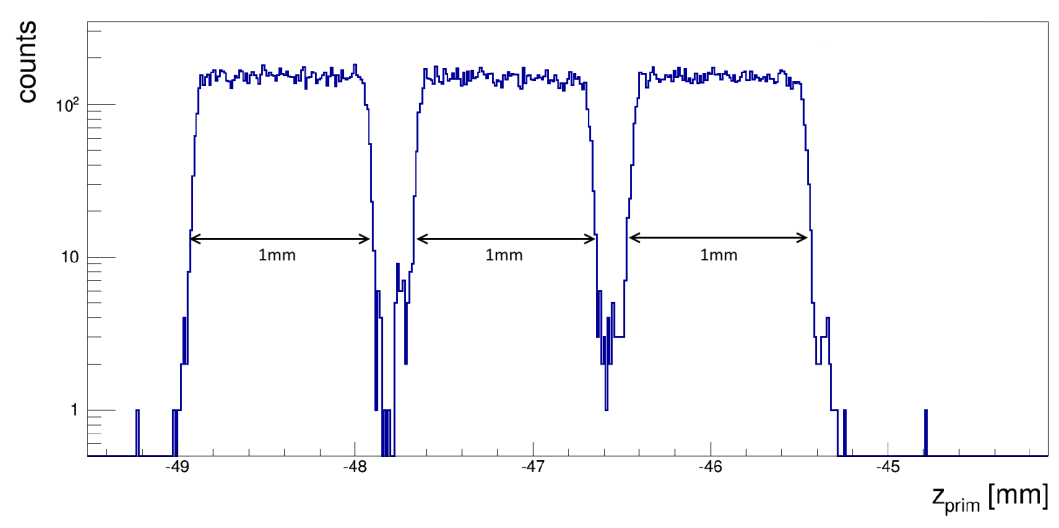

The track matching between VD and TPC was done using the following algorithm: at first, tracks were refitted to the VD primary vertex (for primary tracks) or VD clusters from a given station (for secondary tracks) and then interpolated to other VD stations, and the matching clusters were collected. Finally, the whole track was refitted using the Kalman filter algorithm.

\subsection{Reconstruction of the $D^{0}+\overline{D^{0}}$ Signal}

The SAVD tracks matched to TPC tracks were used to search for the $D^{0}+\overline{D^{0}}$ signal. In the current analysis, particle identification of the daughter particles was not used, however in future it will be used and it is expected to reduce the combinatorial background by factor of 5. Each SAVD track was paired with another SAVD track and was assumed to be either a kaon or pion. Thus, each pair contributed twice in the combinatorial invariant mass distribution. The combinatorial background was several orders of magnitude higher than the $D^{0}+\overline{D^{0}}$ signal due to the low yield of charm particles. In order to reduce the large background, four cuts were applied. The cut values were chosen to maximize the Signal to Noise Ratio (SNR) of the reconstructed $D^{0}+\overline{D^{0}}$ peak and were determined from simulations [33, 34]. These cuts were:

- track transverse momentum $p_{T}>0.34 \mathrm{GeV} / c$;

- track impact parameter $d>34 \mu \mathrm{m}$;

- longitudinal distance between the $D^{0}$ decay candidate and the interaction point $V_{z}>475 \mu \mathrm{m}$;

- impact parameter D of the back-extrapolated $D^{0}$ candidate momentum vector $\mathrm{D}<21 \mu \mathrm{m}$.

Note, that the last three cuts were based on information delivered by the SAVD.

\subsection{Open Charm Measurements with the SAVD}

The SAVD was installed as part of the NA61/SHINE facility and used in 2016 during $\mathrm{Pb}+\mathrm{Pb}$ test run at $150 \mathrm{~A} \mathrm{GeV/c}$ beam momentum. An exploratory set of data was collected and analyzed 
[41, 42]. Figure 9 shows the invariant mass distribution of unlike charge daughter candidates with the applied cuts. One observes a peak emerging at $1.82 \mathrm{GeV} / \mathrm{c}^{2}$, which is considered as an indication of $D^{0}+\overline{D^{0}}$ production. The mean value of the peak is shifted relative to the Particle Data Group (PDG) mass value $1.86 \mathrm{GeV} / \mathrm{c}^{2}$ due to the poorly calibrated TPC data.

After the successful performance of the SAVD in 2016, the devise was used during the $\mathrm{Xe}+\mathrm{La}$ data taking in 2017 and $\mathrm{Pb}+\mathrm{Pb}$ data taking in 2018 and large statistics dataset were taken. This data is now being analysed.

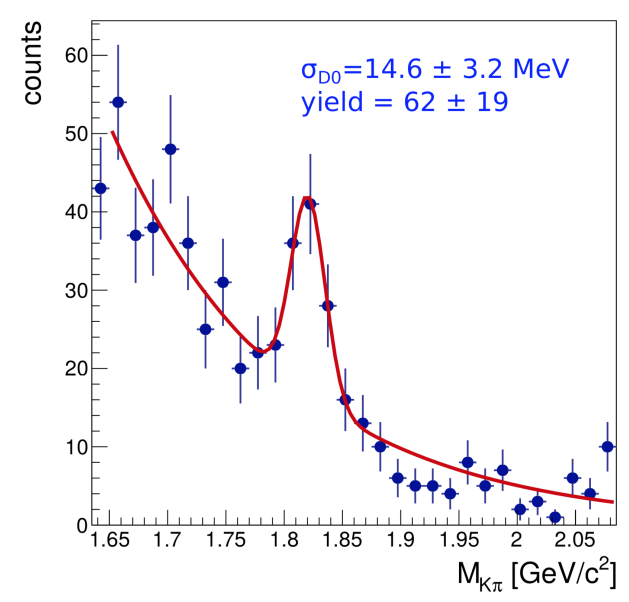

Figure 9: Invariant mass distribution of unlike charge sign $\pi, K$ decay track candidates for $\mathrm{Pb}+\mathrm{Pb}$ collisions at $150 \mathrm{~A}$ $\mathrm{GeV} / c$. The invariant mass distribution was fitted (red line) using an exponential function to describe the background and a Gaussian to describe the $D^{0}+\overline{D^{0}}$ signal contribution. The indicated errors are statistical only.

\section{Proposed Open Charm Measurements after CERN Long Shutdown 2}

Looking forward, the NA61/SHINE experiment will be upgraded during the CERN long shutdown from 2019-2020 to increase the data taking rate from $80 \mathrm{~Hz}$ to $1 \mathrm{kHz}$ [1]. The upgraded VD will be based on the Monolithic Active Pixel sensors (ALPIDE) developed for the ALICE ITS [43] and will have larger acceptance for each station (see Figure 10). The proposed program will allow performing systematic studies of $D^{0}, \bar{D}^{0}, D^{+}$, and $D^{-}$production. This study will provide the total $c \bar{c}$ yield in central $\mathrm{Pb}+\mathrm{Pb}$ collisions needed to investigate the mechanism of charm production. Moreover, the data will allow establishing the centrality dependence of $\langle c \bar{c}\rangle$ in $\mathrm{Pb}+\mathrm{Pb}$ collisions at $150 \mathrm{~A} \mathrm{GeV/c}$ and thus address the question of how the formation of QGP impacts $J / \psi$ production.
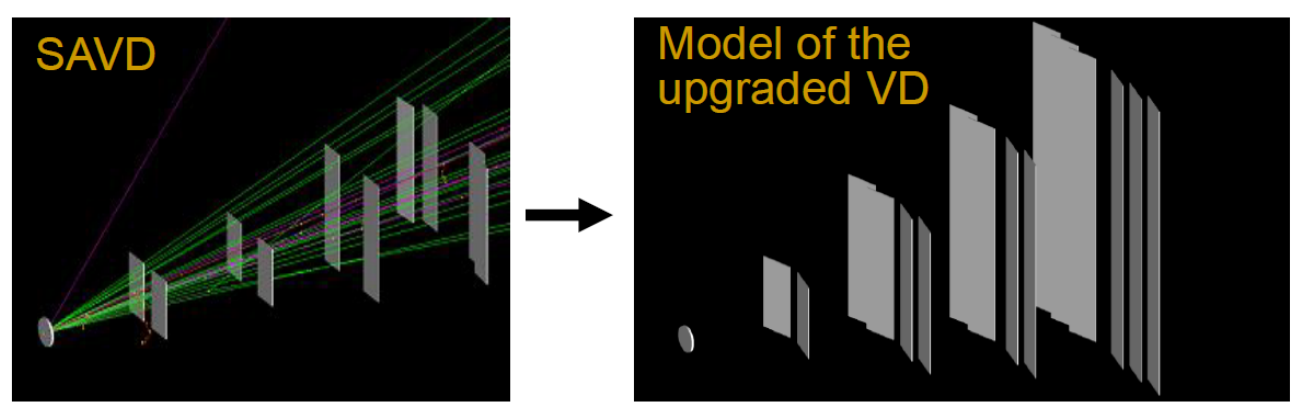

Figure 10: GEANT4 visualization of the current SAVD (left) and the proposed version of the upgraded Vertex Detector (right). 
The simulations performed using AMPT event generator as input show that about 13\% (three times better than for the SAVD) of all $D^{0}+\overline{D^{0}}$ in the $\pi, K$ decay channel can be registered by the upgraded Vertex Detector and pass background suppression and quality cuts (see Figure 11) and about $9 \%$ of all $D^{+}+D^{-}$in the $2 \pi, K$ decay channel (see Figure 12). The total uncertainty of $\left\langle D^{0}\right\rangle$ and $\left\langle\overline{D^{0}}\right\rangle$ is expected to be about $10 \%$ and is dominated by systematic uncertainty.
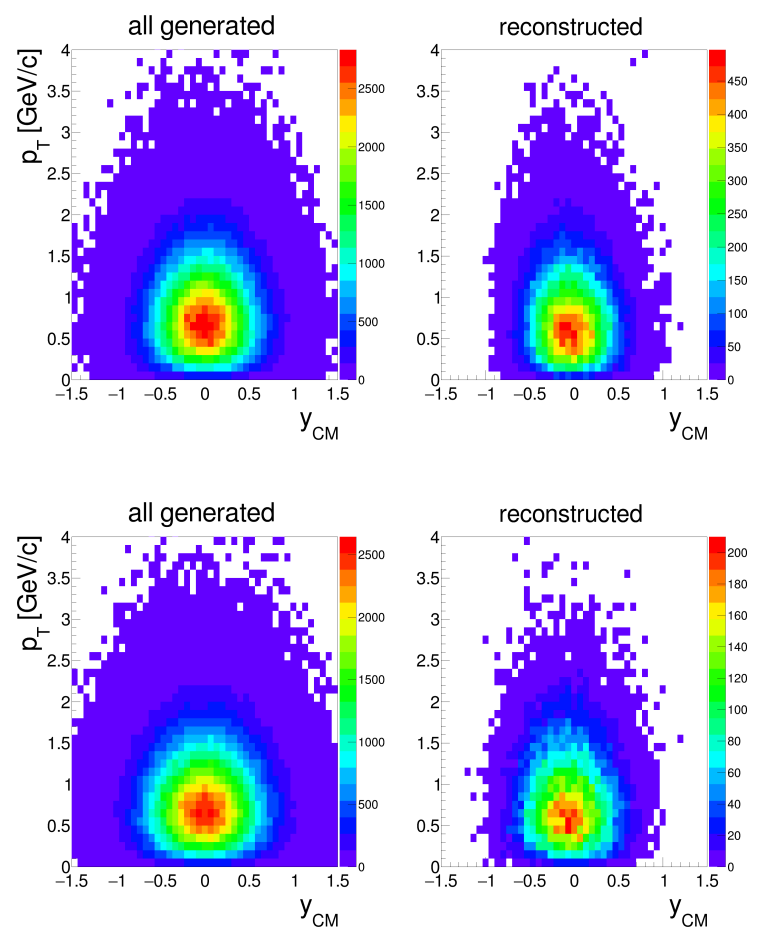

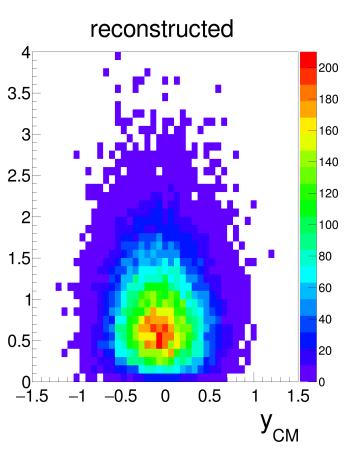

Figure 11: AMPT simulation of transverse momentum and rapidity distributions of $D^{0}+\overline{D^{0}}$ mesons produced in central $\mathrm{Pb}+\mathrm{Pb}$ collisions at $150 \mathrm{~A} \mathrm{GeV/c}$ corresponding to $500 \mathrm{M}$ recorded events. Left: all produced $D^{0}+\overline{D^{0}}$ mesons. Right: $D^{0}+\overline{D^{0}}$ mesons in the $\pi, K$ decay channel and both decay products registered by the upgraded VD and the TPCs and passing background suppression and quality cuts.

Figure 12: AMPT model simulation of transverse momentum and rapidity distributions of $D^{+}+D^{-}$ mesons produced in central $\mathrm{Pb}+\mathrm{Pb}$ collisions at $150 \mathrm{~A} \mathrm{GeV} / c$ corresponding to $500 \mathrm{M}$ recorded events. Left: all produced $D^{+}+D^{-}$mesons. Right: $D^{+}+$ $D^{-}$mesons in the $2 \pi, K$ decay channel and all decay products registered by the upgraded VD and the TPCs and passing background suppression and quality cuts.

The data taking plan related to the open charm measurements in 2021-2023 is shown in Table 1. The estimates for open charm yields were made assuming that the mean multiplicity of charm hadrons is proportional to the number of collisions and using yields calculated for central $\mathrm{Pb}+\mathrm{Pb}$ collisions within the Hadron String Dynamics (HSD) model [3]. In total, 500M minimum bias $\mathrm{Pb}+\mathrm{Pb}$ events are expected to be collected at the top SPS energy. Table 2 lists the expected number of charm mesons in centrality selected $\mathrm{Pb}+\mathrm{Pb}$ collisions at $150 \mathrm{~A} \mathrm{GeV} / c$. These collected data will allow to start the detailed research programme to establish the collision energy dependence of $\langle c \bar{c}\rangle$ at SPS energies and address the question of how the onset of deconfinement impacts charm production.

Table 1: The NA61/SHINE data taking plan for the open charm measurements.

$\begin{array}{ccccc}\text { Year } & \text { Reaction } & \text { Events } & D^{0}+\bar{D}^{0} & D^{+}+D^{-} \\ 2021 & \mathrm{~Pb}+\mathrm{Pb} 150 A \mathrm{GeV} / c & 250 \mathrm{M} & 38 \mathrm{k} & 23 \mathrm{k} \\ 2022 & \mathrm{~Pb}+\mathrm{Pb} 150 A \mathrm{GeV} / c & 250 \mathrm{M} & 38 \mathrm{k} & 23 \mathrm{k} \\ 2023 & \mathrm{~Pb}+\mathrm{Pb} \mathrm{40A} \mathrm{GeV/c} & 250 \mathrm{M} & 3.6 \mathrm{k} & 2.1 \mathrm{k}\end{array}$

\section{Acknowledgements}

The work was supported by the Polish National Center for Science Grant 2014/15/B/ST2/ 
Table 2: Expected number of charm mesons in different centrality $(0-10 \%, 10-20 \%, 20-30 \%, 30-60 \%$, 60$90 \%$, and $0-90 \%$ ) selected $\mathrm{Pb}+\mathrm{Pb}$ collisions at $150 \mathrm{~A} \mathrm{GeV} / \mathrm{c}$ assuming $500 \mathrm{M}$ minimum bias events recorded in 2022 and 2023; see the text for detail. The mean number of wounded nucleons $\langle W\rangle$ calculated within the wounded nucleon model is also given, as well as the mean number of binary collisions $\left\langle N_{C O L L}\right\rangle$.

$\begin{array}{ccccccc} & \mathbf{0 - 1 0} \% & \mathbf{1 0 - 2 0} \% & \mathbf{2 0 - 3 0} \% & \mathbf{3 0 - 6 0} \% & \mathbf{6 0 - 9 0} \% & \mathbf{0 - 9 0} \% \\ D^{0}+\bar{D}^{0} & 31 \mathrm{k} & 20 \mathrm{k} & 11 \mathrm{k} & 13 \mathrm{k} & 1.3 \mathrm{k} & 76 \mathrm{k} \\ D^{+}+D^{-} & 19 \mathrm{k} & 12 \mathrm{k} & 7 \mathrm{k} & 8 \mathrm{k} & 0.8 \mathrm{k} & 46 \mathrm{k} \\ \langle W\rangle & 327 & 223 & 159 & 69 & 11 & 105 \\ \left\langle N_{C O L L}\right\rangle & 769 & 443 & 292 & 102 & 10 & 203\end{array}$

02537, the Polish Ministry of Science and Higher Education DSC Grant 7150/E-338/M/2018 No. K/DSC/004993, the Polish National Center for Science Grant 2018/29/N/ST2/02595, the Russian Science Foundation research Grant 16-12-10176, and partially by the National Science Centre, Grant 2015/18 /M /ST2 /00125 and the Ministry of Science and Higher Education Grant DIR/WK/2016/2017/10-1.

\section{References}

[1] A. Aduszkiewicz et al. (NA61/SHINE Collaboration), CERN-SPSC-2018-008 / SPSC-P-330-ADD-10. Available online: http://cds.cern.ch/record/2309890.

[2] O. Linnyk, E. L. Bratkovskaya and W. Cassing, Int. J. Mod. Phys. E17 (2008) 1367.

[3] O. Linnyk, E. L. Bratkovskaya and W. Cassing, Int. J. Mod. Phys. E17 (2008) 1367, doi:10.1142/S0218301308010507.

[4] T. Song, private communication.

[5] R. Gavai, et al., Int. J. Mod. Phys. A 10 (1995) 2999, doi:10.1142/S0217751X95001431.

[6] P. Braun-Munzinger, and J. Stachel, Phys. Lett. B 490 (2000) 196, doi:10.1016/S0370-2693(00)00991-6

[7] P. Levai, et al., J. Phys. G 27 (2001) 703, doi:10.1088/0954-3899/27/3/357.

[8] A. P. Kostyuk et al., Phys. Lett. B 531 (2002) 195, doi:10.1016/S0370-2693(02)01488-0.

[9] Cassing, W. and Bratkovskaya, E.L. Nucl. Phys. A 831 (2009) 215, doi:10.1016/j.nuclphysa.2009.09.007.

[10] T. Matsui and H. Satz, 1986, Phys. Lett. B 178, 416, doi:10.1016/0370-2693(86)91404-8.

[11] Satz, H., High Energy Phys. 2013 (2013) 242918, doi:10.1155/2013/242918.

[12] Muller, B. Phys. Scripta T 158 (2013) 014004, doi:10.1088/0031-8949/2013/T158/014004.

[13] R. V. Poberezhnyuk, M. Gazdzicki, and M. I. Gorenstein, 2017, Acta Phys. Polon. B 48, 1461, doi:10.5506/APhysPolB.48.1461.

[14] J. Rafelski and B. Muller, 1982, Phys. Rev. Lett. 48, 1066, doi:10.1103/PhysRevLett.48.1066.

[15] C. Alt et al. (NA49 Collaboration), Phys. Rev. C 73 (2006) 034910 doi:10.1103/PhysRevC.73.034910 [nucl-ex/0507031]. 
[16] M. Masera et al. (NA50 Collaboration), 2001, J. Phys. G: Part. Phys. 27, 677, doi:10.1088/0954-3899/27/3/353.

[17] Scomparin, E. et al. (NA60 Collaboration), Nucl. Phys. A 830 (2009) 239C, doi:10.1016/j.nuclphysa.2009.10.020.

[18] Heinz, U.W.; Jacob, M. Evidence for a new state of matter: An Assessment of the results from the CERN lead beam program. Available online: https://arxiv.org/abs/nucl-th/0002042.

[19] A. N. Petridis, 1996, Phys. Rev. C 54, 848, doi:10.1103/PhysRevC.54.848.

[20] E. Meninno et al. (ALICE Collaboration), 2017, EPJ Web Conf. 137, 06018, doi:10.1051/epjconf/201713706018.

[21] G. W. S. Hou et al. (ATLAS, CMS Collaboration), 2016, PoS CHARM2016, 088, doi:10.22323/1.289.0088.

[22] M. Simko et al. (STAR Collaboration), 2017, J. Phys. Conf. Ser. 832 no. 1, 012028 , doi:10.1088/1742-6596/832/1/012028.

[23] K. Nagashima et al. (PHENIX Collaboration), 2017, Nucl. Phys. A967, 644-647, doi:10.1016/j.nuclphysa.2017.06.038.

[24] G. Odyniec, 2013, J. Phys. Conf. Ser. 455, 012037, doi:10.1088/1742-6596/455/1/012037.

[25] C. Yang et al. (STAR Collaboration), 2017, Nucl. Phys. A967, 800, doi:10.1016/j.nuclphysa.2017.05.042.

[26] K. C. Meehan et al. (STAR Collaboration), 2016, Nucl. Phys. A 956, 878, doi:10.1016/j.nuclphysa.2016.04.016.

[27] Kekelidze, V.; Kovalenko, A.; Lednicky, R.; Matveev, V.; Meshkov, I.; Sorin, A.; Trubnikov, G., 2017, Nucl. Phys. A967, 884, doi:10.1016/j.nuclphysa.2017.06.031.

[28] H. Sako et al., (J-PARC Heavy-Ion Collaboration), 2016, Nucl. Phys. A 956, 850, doi:10.1016/j.nuclphysa.2016.03.030.

[29] B. Friman, C. Hohne, J. Knoll, S. Leupold, J. Randrup, R. Rapp, and P. Senger, 2011, Lect. Notes Phys.814, 1, doi:10.1007/978-3-642-13293-3.

[30] N. Abgrall et al. (NA61 Collaboration), 2014, JINST 9, P06005, doi:10.1088/1748-0221/9/06/P06005.

[31] Afanasev, S. et al. (NA49 Collaboration), Nucl. Instrum. Meth. A 430 (1999) 210, doi:10.1016/S0168-9002(99)00239-9.

[32] Aduszkiewicz, A. et al. (NA61 Collaboration), CERN-SPSC-2015-038 / SPSC-P-330-ADD-8. Available online: http://cds.cern.ch/record/2059811.

[33] Ali, Y.; Staszel, P.; Marcinek, A.; Brzychczyk, J.; Planeta, R., Acta Phys. Polon. B 44 (2013) no.10, 2019, doi:10.5506/APhysPolB.44.2019.

[34] Ali, Y. et al. (NA61 Collaboration), J. Phys. Conf. Ser. 509 (2014) 012083, doi:10.1088/1742-6596/509/1/012083.

[35] Z.-W. Lin et al., 2005, Phys. Rev. C72, 064901, doi:10.1103/PhysRevC.72.064901.

[36] M. Deveaux et al., 2011, Journal of Instrumentation 6(02), C02004, doi:10.1088/1748-0221/6/02/C02004. 
[37] B. Abelev et al. (ALICE Collaboration), 2014, J.Phys.G41, 087002, doi:10.1088/0954-3899/41/8/087002.

[38] J. Michel et al., 2011, IEEETNS 58, 1745, doi:10.1109/TNS.2011.2141150.

[39] P. Klaus et al., 2016, JINST 11, C03046, doi:10.1088/1748-0221/11/03/C03046.

[40] A. Merzlaya et al., 2017, Journal of Physics: Conference Series 798(1), 012072, doi:10.1088/1742-6596/798/1/012072.

[41] A. Merzlaya et al., KnowledgeE Energy \& Physics 3, 154 (2018), doi:10.18502/ken.v3i1.1738.

[42] A. Merzlaya et al., Universe 5 (2019) no.1, 14, doi:10.3390/universe5010014

[43] G. Aglieri et al. (ALICE Collaboration), 2013, JINST 8, C12041, doi:10.1088/1748-0221/8/12/C12041. 